\title{
Induction of Biologically Active Antibodies in Mice, Rabbits, and Monkeys by Plasmodium falciparum EBA-175 Region II DNA vaccine
}

\author{
B. Kim Lee Sim, ${ }^{1}$ David L. Narum, ${ }^{1}$ Hong Liang, ${ }^{1}$ Steven R. Fuhrmann, ${ }^{1}$ Nicanor Obaldia III, ${ }^{2}$ \\ Robert Gramzinski, ${ }^{4}$ Joao Aguiar, ${ }^{4}$ J. David Haynes, ${ }^{3}$ J. Kathleen Moch, ${ }^{4}$ and Stephen L. Hoffman ${ }^{4}$ \\ ${ }^{1}$ EntreMed, Inc., Department of Molecular Biology, Rockville, MD, USA; \\ ${ }^{2}$ Promed/Gorgas Memorial Laboratory, Panama City, Panama \\ ${ }^{3}$ Department of Immunology, Walter Reed Army Institute of Research, Washington, DC, USA \\ ${ }^{4}$ Malaria Program, Naval Medical Research Center, Silver Spring, MD, USA \\ Accepted January 29, 2001
}

\begin{abstract}
Background: Plasmodium falciparum merozoites bind to and invade human erythrocytes via specific erythrocyte receptors. This establishes the erythrocytic stage of the parasite life cycle that causes clinical disease resulting in 2-3 million deaths per year. We tested the hypothesis that a Plasmodium falciparum ligand, EBA-175 region II (RII), which binds its erythrocyte receptor glycophorin A during invasion, can be used as an immunogen to induce antibodies that block the binding of RII to erythrocytes and thereby inhibit parasite invasion of erythrocytes. Accordingly, we immunized mice, rabbits, and monkeys with DNA plasmids that encoded the 616 amino acid RII.

Materials and Methods: DNA vaccine plasmids that targeted the secretion of recombinant RII protein with and without the universal T-cell helper epitopes P2P30 were used to immunize mice, rabbits, and Aotus monkeys. RII specific antibodies were assessed by IFA, ELISA, blocking of native $\left[{ }^{35} \mathrm{~S}\right]$ labeled EBA-175 binding to human erythrocytes, and growth inhibition assays, all in vitro.
\end{abstract}

Results: The RII DNA plasmids were highly immunogenic as measured by ELISA and IFA. The anti-RII antibodies blocked the binding of native EBA-175 to erythrocytes, and rosetting of erythrocytes on COS-7 cells expressing RII. Most important, murine and rabbit antiRII antibodies inhibited the invasion of merozoites into erythrocytes. We immunized nonhuman primates and showed that the RII-DNA plasmids were immunogenic and well tolerated in these monkeys. Monkeys were challenged with parasitized erythrocytes; one of three monkeys that received RII DNA plasmid was protected from fulminant disease. After challenge with live parasites, anti-RII antibody titers were boosted in the immunized monkeys.

Conclusions: By proving the hypothesis that anti-RII antibodies can block merozoite invasion of erythrocytes, these studies pave the way for the clinical evaluation of EBA-175 as a receptor-blockade vaccine.

\section{Introduction}

Invasion of erythrocytes by malaria parasites requires parasite ligands that interact with specific erythrocyte receptors. The $175 \mathrm{kDa}$ Plasmodium falciparum erythrocyte binding protein (EBA-175) mediates invasion of erythrocytes by binding to erythrocytes $(1,2)$. The domain of EBA-175 that binds erythrocytes was identified as region II (RII) using truncated portions of EBA-175 expressed on COS-7 cells (3). RII, which contains a duplicated cysteine-rich motif, and native EBA-175 bind specifically to glycophorin A on the erythrocyte membrane. This binding is dependent on sialic acids on glycophorin A because metabolically labeled native EBA-175 fail to bind desialated glycophorin A as well as neuraminidase treated erythrocytes (3).

Address correspondence and reprint requests to: Dr. B. Kim Lee Sim, EntreMed, Inc., 9640 Medical Center Drive, Rockville, MD 20850, USA. Phone: (301) 738-2485. Fax: (301) 217-9594. E-mail: kims@entremed.com
Sequence analysis of RII shows complete conservation of cysteine residues in more than 30 laboratory strains as well as wild isolates, indicating that the tertiary structure of RII is probably required for function (4) (Liang, unpublished data). At the amino acid level, strict conservation of sequences between cysteines occurs, with the exception of 5 to 13 specific residues scattered throughout RII. By examining targeted surface expression of RII on the surface of COS-7 cells, we found that these few changes limited to specific residues did not affect the binding function of RII. Furthermore, the sialic acid binding specificity was conserved because none bound to neuraminidase-treated erythrocytes (4). These results further emphasize the importance of assessing EBA-175 RII as a candidate for receptorblockade vaccines.

We hypothesized that antibodies raised against RII would interfere with the ability of merozoites to bind to erythrocytes during invasion, thus inhibiting invasion. To test this hypothesis, we immunized mice and rabbits with DNA plasmids containing the entire 
RII gene sequence. We report that the RII plasmids were highly immunogenic, and that the antibodies generated were potent at inhibiting merozoite invasion of erythrocytes. We also assessed the safety, immunogenicity, and protective efficacy of the most promising immunogen studied, in Aotus monkeys. The results of these studies support the further development of EBA-175 as a receptor-blockade vaccine.

\section{Materials and Methods}

\section{Parasites}

Plasmodium falciparum parasites strains 3D7 (clone of NF54, Amsterdam Airport, chloroquine sensitive strain used in human trials) and FVO (Uganda, Aotus adapted strain) were cultured as previously reported (5). A thermal-cycler incubator maintained parasite synchrony (Haynes and Moch, manuscript in preparation), and schizonts were purified on Percoll density gradients (6). The FVO parasites were metabolically labeled with TRAN ${ }^{35}$ S-LABELTM (ICN Radiochemicals, Irvine, CA) as previously described (3). Parasite culture supernatants containing labeled EBA-175 were stored at $-70^{\circ} \mathrm{C}$.

\section{Construction of EBA-175 RII DNA Vaccines}

P. falciparum EBA-175 RII (amino acids 145-760, 1848bp for either FVO or 3D7) was amplified using Vent DNA polymerase (New England BioLabs, Beverly, MA) from 100 ng of genomic DNA using the forward primer 5'ATCGAGATCTGGAAGAAATACTTCATCT3'. Reverse primers 5'ATCGAGATCTTTACGAAGTTTGTTCATTATT3' or 5'ATCGAGATCTCGAAGTTTGTTCATTATT3' were used for cloning into the expression plasmid vectors VR1020 and VR1050, respectively $(7,8)$. These plasmid vectors use the human cytomegalovirus promoter and intron A, human tissue plasminogen activator, as the secretory signal and the bovine growth hormone transcriptional terminator/polyadenylation signal. To enhance immunogenicity, the expression plasmid vector (VR1050) contained the gene sequences encoding the universal T-epitopes from tetanus toxin P2P30 (9), which were located $5^{\prime}$ to the RII gene in the DNA vaccine. The clones were selected for correct orientation by PCR and sequence-verified. Human melanoma cells (UM449) were transiently transfected with the plasmids VR 1020 and VR 1050 containing RII insert with Lipofectamine ${ }^{\mathrm{TM}}$ following the manufacture's protocol (Life Technologies, Gaithersburg, MD). Secretion of RII protein in culture supernatants was confirmed by Western blot. The resultant plasmid vaccines for FVO RII inserted into VR 1020 and VR1050 are VR2526 (pFVORII) and VR2527 (pFVORII/P2P30), respectively. Purity gauged by UV spectroscopy $(260 \mathrm{~nm} / 280 \mathrm{~nm})$ was between 1.70 and 1.90; agarose gel electrophoresis showed predominately supercoiled plasmid, and endotoxin levels detected using the Limulus amebocyte assay were less than $10 \mathrm{EU} / \mathrm{mg}$ plasmid DNA.

\section{Animals and Immunizations}

All animal studies were done in compliance with protocols approved by Animal Care and Use Committees. Groups of $5 \mathrm{~A} / \mathrm{J}, \mathrm{Balb} / \mathrm{c}, \mathrm{CAF} / \mathrm{J}, \mathrm{C} 57 \mathrm{Bl} / 6$, and $\mathrm{DBA} / 2 \mathrm{~J}$ mice were inoculated intradermally with a 29-gauge needle at two sites in the tail, $50 \mu \mathrm{g}$ per site with VR 1020 (empty vector), pFVORII, and pFVORII/P2P30 in $50 \mu \mathrm{l}$ PBS. The mice were inoculated on days 0,21 , and 42 and bled on days $-1,14$, 35, and 56. Pooled sera were stored at $-70^{\circ} \mathrm{C}$ until used. Rabbits received a total of $3 \mathrm{mg}$ of DNA vaccine at $1 \mathrm{mg} / \mathrm{mL}$ in PBS and administered as follows: 1) bilaterally into each quadriceps muscle $(2 \times$ $500 \mu \mathrm{l}), 2)$ bilaterally into each gluteus maximus muscle $(2 \times 500 \mu \mathrm{l})$, and 3$)$ intradermal injections $(10 \times 100 \mu \mathrm{l})$ across the back. Both male and female Aotus lemurinus lemurinus (karyotype VII or IX) monkeys (10) weighing between 800-900 grams were used in our studies. These monkeys were maintained in the animal facility of the Gorgas Memorial Laboratory in Panama City, Republic of Panama, cared for as previously described (11) and in accordance with the Guide for the Care and Use of Laboratory Animals of the National Research Council, 1996. Intradermal delivery of DNA vaccines was performed under light sedation with Ketamine at $20 \mathrm{mg} / \mathrm{kg}$ intramuscularly, using a $1 \mathrm{~mL}$ insulin syringe with a fused 29-gauge 0.5-inch needle (BectonDickenson, Franklin Lakes, NJ). Monkeys received a total of $500 \mu \mathrm{g}$ of plasmid DNA in saline in a series of four immunizations at weeks $0,3,6$, and 47 on the lower back on six different sites. The maximal volume administered in any one site was $100 \mu \mathrm{l}$.

Enzyme-linked Immunosorbent Assay (ELISA)

Serum antibodies were assayed essentially as described (12). The capture antigen was a baculovirus recombinant EBA-175 RII protein (13). Goat antihuman IgG (Promega, Madison, WI), which crossreacted with Aotus IgG, was used as the secondary antibody for the ELISA. Substrate was added (100 $\mu \mathrm{l} /$ well) and incubated for $1 \mathrm{hr}$ for rabbit or $30 \mathrm{~min}$ for Aotus sera (Pierce, Rockford, IL). The optical density at $635 \mathrm{~nm}$ was determined using a Molecular Devices Spectra Max 250 (Sunnyvale, CA). The ELISA results are reported as the interpolated reciprocal dilution estimated to give an optical density (OD) of 0.5.

\section{Indirect Immunofluorescence Assay (IFA)}

Mature erythrocytic stages of FVO and 3D7 strains of $P$. falciparum cultured in vitro were used to prepare thin films of parasitized blood. Parasitized erythrocytes were washed three times in PBS containing $2 \%$ BSA, dispensed in slide wells, left to air-dry, and then frozen at $-70^{\circ} \mathrm{C}$. After slides were thawed in a dehumidifier, duplicate wells were overlaid with $15 \mu \mathrm{l}$ of the test and control antibody dilutions and incubated in a moist chamber at $37^{\circ} \mathrm{C}$ for $60 \mathrm{~min}$. Fluorescence detection was done with the appropriate secondary antibodies diluted in PBS containing 
$\mathbf{0 . 0 0 5 \%}$ Evans blue. When testing mice, rabbit, and Aotus monkey sera, FITC-labeled goat anti-mouse IgG (Becton Dickinson, San Jose, CA), FITC-labeled goat anti-rabbit IgG (Kirkegaard \& Perry Laboratories, Inc., Gaithersburg, MD), and FITC-labeled goat anti-human IgG (ICN Biomedical, Costa Mesa, CA) were used respectively. FITC-labeled goat antihuman IgG, which cross-reacted with Aotus IgG, was used for the IFA. Slides were examined under an Olympus UV microscope. End-point titers were determined as the last dilution above the background, in which fluorescent parasites were observed. Background fluorescence was determined using preimmunization sera for each immunized group.

\section{Inhibition of Merozoite Invasion in Vitro}

For the analysis of antibody mediated blockade (growth or invasion inhibition assays, GIA), triplicate wells in 48-well plates were prepared as for static cultures (5), but these were kept suspended on a rotator platform (Haynes and Moch, in preparation). Briefly, synchronized cultures of P. falciparum schizont-infected erythrocytes were mixed with test or control sera (after heat inactivation and adsorption three times with human erythrocytes) so that final sera concentration (v/v) was $10 \%$ (mouse sera) or $20 \%$ (rabbit sera) and the final hematocrit was $1.5 \%$ (mouse sera) or $6 \%$ (rabbit sera). Pooled A/J mouse sera were used to increase the amount of material for testing. A final $10 \%$ heat-inactivated normal human serum was included in all microcultures with bicarbonate-containing RPMI 1640 and gassed with 5\% $\mathrm{O}_{2} 5 \% \mathrm{CO}_{2}$. After a single cycle of merozoite invasion, Giemsa stained thin smears were prepared. Parasitemias were determined by counting the number of parasites in 5000 RBCs. The percentage inhibition was calculated from the mean parasitemias of triplicate test and control wells as $100 \times($ control-test $) /$ control.

\section{Inhibition of Erythrocytes Binding to COS-7 Cells} Expressing RII on the Surface

COS-7 cells were transfected with the RII plasmid DNA in 6-well culture dishes as described (3). Pooled sera from immunization groups were diluted in DMEM with 10\% FBS and $1 \mathrm{~mL}$ was used to replace media of COS-7 cell monolayers. The monolayers with sera were incubated for 1 hour at $37^{\circ} \mathrm{C}$, followed by addition of $10 \mu \mathrm{l}$ packed $\left(1 \times 10^{8}\right)$ human erythrocytes. The dish was swirled gently and incubated for an additional $\mathrm{l} \mathrm{hr}$ at $37^{\circ} \mathrm{C}$. Nonadherent erythrocytes were washed off monolayers by gentle removal and replacement of culture media/10\% FBS. The number of COS-7 cells with rosettes of erythrocytes were scored using an inverted microscope. A COS-7 cell with a rosette of erythrocytes was defined as more than 10 erythrocytes adhering to a single cell.

Blocking of Native EBA-175 Binding to Erythrocytes

$\left[{ }^{35}\right.$ S]-metabolically labeled EBA-175 from parasite culture supernatants was used for binding assays as previously described (3). The blocking of native $\left[{ }^{35} \mathrm{~S}\right]-\mathrm{EBA}-175$ binding to erythrocytes by rabbit serum was calculated as the percentage of blocking relative to the control at a 1:10 dilution. For Aotus monkeys, the blocking of binding was reported as the reciprocal dilution resulting in 50\% inhibition relative to the control. The "control" was the counts from $\left[{ }^{35} \mathrm{~S}\right]-\mathrm{EBA}-175$ bound to erythrocytes in the presence of normal rabbit or Aotus sera. The counts due to background were subtracted from all samples.

\section{Aotus Challenge Studies}

Aotus monkeys were challenged with $1 \times 10^{4} P$. falciparum (FVO) infected erythrocytes as follows: on day 0 , the donor monkey was bled via the ear vein and its parasitemia calculated as parasites $/ \mu \mathrm{L}$. The monkey was then bled via the saphenous vein using a $1 \mathrm{~mL}$ syringe containing acidified citrate dextrose (Red Cross, Rockville, MD). A precalculated amount of blood was withdrawn and mixed, immediately on withdrawal from the animal, with a premeasured amount of RPMI $\left(37^{\circ} \mathrm{C}\right)$ resulting in a cell suspension containing 10,000 P. falciparum-infected Aotus erythrocytes per mL. This preparation was gently swirled as a syringe for each of the nine monkeys was filled. Each monkey received $1 \mathrm{~mL}$ of the cell suspension, administered intravenous via the saphenous vein. Animals were monitored daily after they were infected for parasites. The daily blood films (thick films only) were prepared from blood taken by lancet from each animal's ear. The films were air-dried and stained with Giemsa stain. Parasitemias were evaluated by the Earle-Perez method. Ten microliters of blood was dispensed onto a measured area on a microscope slide; parasitemias were calculated as "parasites per $\mu \mathrm{l}$ of blood." Twice a week, the animals were bled for hematocrit determination and serum collection. In our study, vaccine failure was defined as 1) parasitized erythrocytes above or equal to 400,000 parasites per $\mu \mathrm{L}$ (approximately 10\%), at which time the monkey was treated with $40 \mathrm{mg}$ mefloquine (single oral dose); 2 ) the hematocrit decreased by $50 \%$ over pre-infection values; or 3) other symptoms such as anorexia or depression appeared. After treatment, the monkeys were monitored twice a week for at least 100 days until there was no sign of parasites in Giemsa-stained thick films and the hematocrits had returned to normal.

\section{Results}

\section{Expression in Vitro of EBA-175 RII}

Human melanoma cells were transfected with plasmids pFVORII, pFVORII/P2P30, p3D7RII, or p3D7RII/P2P30 and expressed RII in culture supernatants (Figure 1). All four DNA vaccine constructs secreted a protein of approximately $70 \mathrm{kDa}$, which was detected by polyclonal antibodies from rabbits immunized with pFVORII/P2P30 (predicted molecular mass of RII is $73.6 \mathrm{kDa}$ ). The secreted proteins from plasmid constructs, which contained the P2P30 


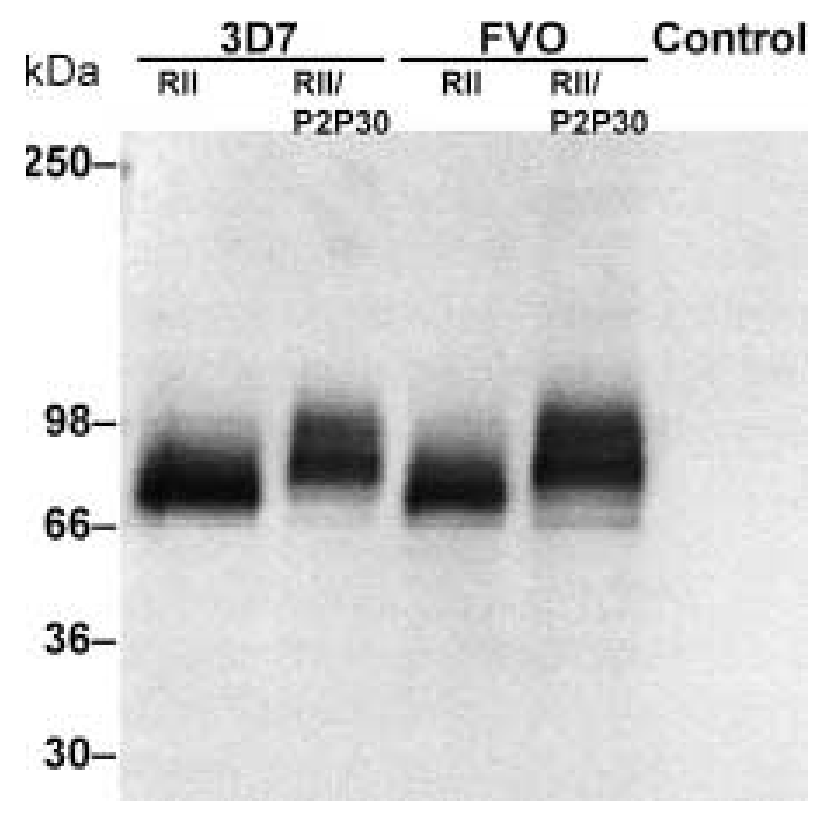

Fig. 1. Western blot of secreted $P$. falciparum 3D7 and FVO RII proteins showing expression in vitro. UM449 cells were transiently transfected with DNA plasmids VR1020 and VR 1050 containing 3D7 and FVO RII, respectively. RII protein was detected by polyclonal rabbit antibodies generated by DNA vaccination. Empty vector control (VRIOZO) is shown. Molecular weight designations are given on the $\mathrm{x}$-axis of the figure.

gene fragment fused to RII, were slightly larger in molecular mass owing to the expression of P2P30 (predicted size of P2P30 is $4.3 \mathrm{kDa}$ ).

Immunogenicity in mice, rabbits, and Aotus monkeys of RII DNA vaccines

All mice immunized with pFVORII and pFVORII/ P2P30 generated antibodies that recognized EBA-175 as detected by IFA against parasitized erythrocytes. The antibody titers by end-point dilution after three immunizations ranged from $1 / 1280$ to $1 / 10240$ and were still detectable 22 weeks after the final immunization (Table 1). In mice, there was indication that inclusion of the P2P30 universal T-helper epitopes enhanced antibody responses.

Immunization of rabbits with pFVORII/P2P30 by subcutaneous and intradermal administration generated significant anti-RII antibody responses. The serum dilution at which the OD was 0.5 by ELISA was 60,000 and 70,000 for the two rabbits. The IFA titer was 10,000 for both.

Aotus monkeys immunized in groups of three also generated anti-RII antibody responses. Analysis of the Aotus sera after each immunization demonstrated that the monkeys that received pFVORII/ P2P30 developed anti-RII antibody titers sooner, and these titers appeared higher through three immunizations, but the differences did not reach the level of statistical significance $(p>0.05)$. The geometric mean ELISA titers for the pFVORII and pFVORII/ P2P30 groups after three immunizations were 200 and 2500 (Figure 2), whereas the corresponding IFA titers were $1: 80$ and 1:1280, respectively. Approximately, a 10-fold enhancement existed with the P2P30 fragment at this point. However, after a complete regimen of four immunizations, the geometric mean titers and individual titers were equivalent for the two different plasmid constructs (Figure 2).

Inhibition of P. falciparum Growth or Invasion in Vitro by Mouse and Rabbit Polyclonal Antibodies

Plasmid DNA vaccination of mice and rabbits with pFVORII/P2P30 produced anti-RII antibodies that inhibited $P$. falciparum FVO growth in vitro. Mouse and rabbit immune sera at a dilution of $1: 10$ and $1: 5$ inhibited growth by $66 \%$ and $43 \%$, respectively, as compared to sera from mice and rabbits that received the empty plasmid (VR1020) DNA control (Table 2).

Inhibition of Rosetting of Erythrocytes to COS-7 Cells Expressing EBA-175 RII on the Cell Surface by Anti-RII Polyclonal Antibodies

Pooled serum from the $\mathrm{A} / \mathrm{J}$ mice and rabbits at $1: 320$ dilution inhibited the binding of erythrocytes to COS7 cells expressing RII by $100 \%$. Aotus sera did not completely inhibit rosetting, but at a dilution of $1: 4$, the sera markedly reduced the number of erythrocytes $(>50 \%)$ per COS-7 cell expressing RII (data not shown). The reciprocal end-point IFA titers against infected

TABLE 1. Titers as determined by IFA against $P$. falciparum infected erythrocytes of pooled sera $(\mathrm{n}=5)$ taken two weeks after the third immunization for 5 strains of mice immunized with RII DNA plasmid constructs

\begin{tabular}{lcccccc}
\hline & \multicolumn{5}{c}{ Reciprocal IFA Titer } \\
\cline { 2 - 6 } CONSTRUCT & DBA/2 & Balb/c & A/J & C57Bl/6 & CAF \\
\hline pFVORII & 2560 & 5120 & 10240 & 2560 & 5120 \\
pFVORII/P2P30 & 2560 & 1280 & 10240 & 2560 & 2560 \\
Control (VR1020) & $<10$ & $<10$ & $<10$ & $<10$ & $<10$ \\
\hline
\end{tabular}




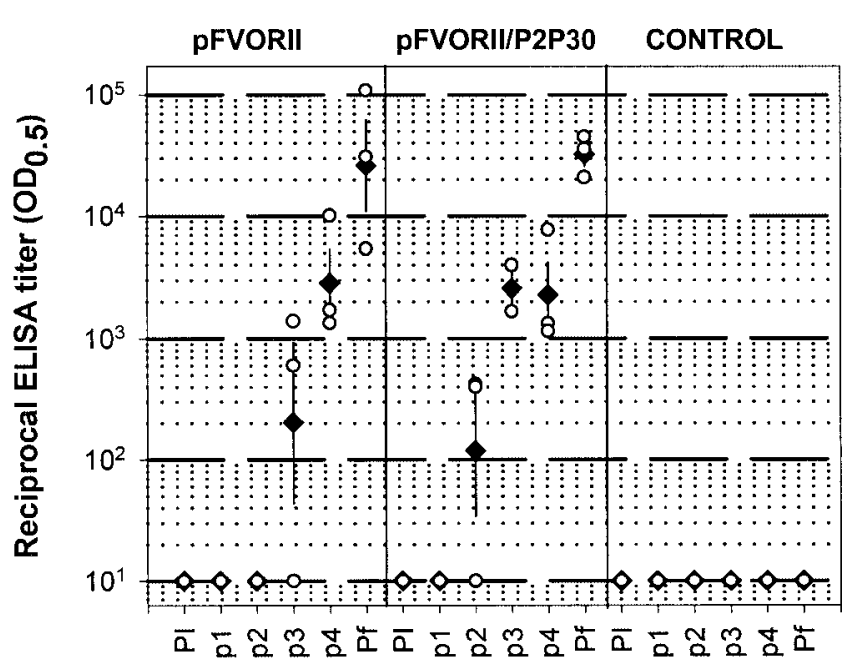

GROUP

Fig. 2. Aotus antibody responses to EBA-175 RII after immunization with DNA plasmids containing RII. Groups of three monkeys were immunized intradermally 4 times at time $0,4,8$, and 24 weeks with pFVORII, pFVORII/P2P30, or VR 1020 (empty plasmid). The Aotus monkeys were challenged with the homologous $P$. falciparum FVO strain two weeks after the $4^{\text {th }}$ immunization and sera were tested from approximately 3 weeks postchallenge. Open circles $(\bullet)$ represent individual reciprocal titers at an ELISA OD of 0.5. Solid diamonds $(\bullet)$ represent reciprocal geometric mean titers at an ELISA OD of 0.5. PI represents pre-immune sera and Pf represents postchallenge sera. Error bars represent standard error of the mean.

erythrocytes for each animal species were consistent with the inhibition of rosetting patterns observed, i.e., A/J, 10,000, rabbit, 20,000, and Aotus, 2500.

Inhibition of Native EBA-175 Binding to Erythrocytes by Anti-RII polyclonal Antibodies

Sera from mice, rabbits, and Aotus monkeys blocked binding of $\left[{ }^{35} \mathrm{~S}\right]$-metabolically labeled $P$. falciparum EBA-175 to erythrocytes. Using sera from A/J

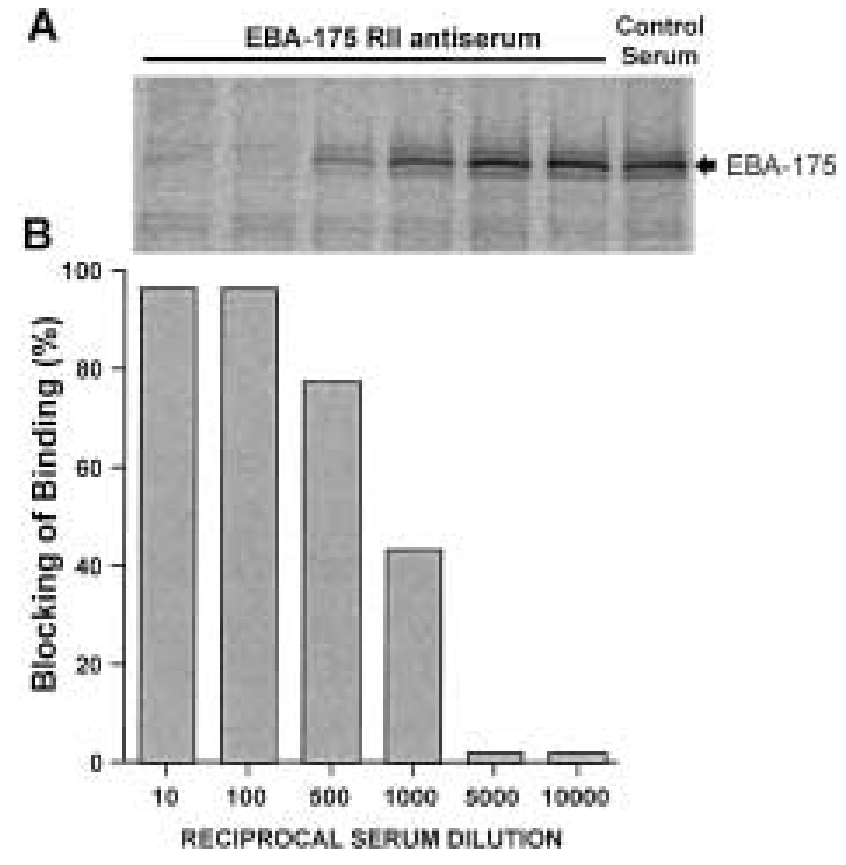

Fig. 3. Rabbit EBA-175 RII specific antibodies block native EBA-175 binding to human erythrocytes. $\left[{ }^{35}\right.$ S]-labeled EBA175 culture supernatants were incubated with a titration of RII specific antibodies and then added to human erythrocytes. Erythrocytes were pelleted as described in Materials and Methods and bound $\left[{ }^{35}\right.$ S]-labeled EBA-175 was eluted and detected by phospho-imaging (A). B shows the percent blocking of native EBA-175 binding to erythrocytes as compared to the control sera at a $1 / 10$ dilution.

mice and rabbits immunized with pFVORII/P2P30, we achieved a $50 \%$ inhibition at a serum dilution of approximately 1:500 for A/J mice (data not shown) and approximately 1:1000 for rabbits (Figure 3) compared to empty vector (VR1020) immunized controls. Most significantly, Aotus monkeys immunized with either pFVORII or pFVORII/P2P30 generated antibodies that blocked EBA-175 binding to human erythrocytes. The individual reciprocal blocking

TABLE 2. Inhibition of $P$. falciparum FVO growth or invasion with anti-EBA-175 RII polyclonal sera in vitro

\begin{tabular}{ccccc}
\hline & \multicolumn{2}{c}{ \%Parasitemia $^{\mathbf{b}}$} & \multicolumn{1}{c}{$\begin{array}{c}\text { Inhibition } \\
\text { (\% of control) }\end{array}$} & Paired t-test \\
\cline { 2 - 5 } Sera $^{\mathrm{a}}$ & Control $^{\mathbf{c}}$ & Experimental & 66 & $<0.01$ \\
Mouse & $0.82 \pm 0.07$ & $0.28 \pm 0.08$ & 43 & $<0.05$ \\
\hline
\end{tabular}

${ }^{a}$ Pooled A/J sera and individual rabbit serum were tested at a dilution of 1:10 and 1:5, respectively for one cycle of invasion in suspension microcultures.

${ }^{\mathrm{b}}$ Values are means \pm standard errors of triplicates.

${ }^{\mathrm{c}}$ Control sera were from mice or a rabbit immunized with empty vector (VR1020). 


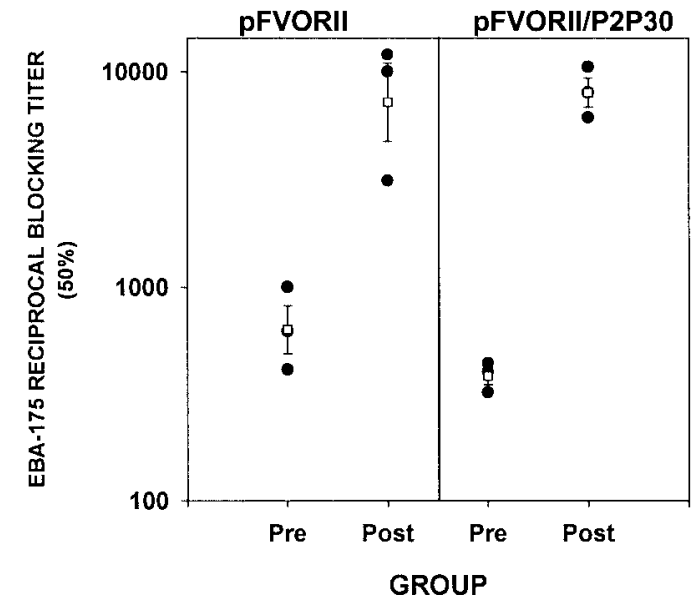

Fig. 4. Blocking of native EBA-175 binding to erythrocytes with sera from Aotus monkeys obtained at pre-challenge and 3 weeks postchallenge with $P$. falciparum FVO strain infected erythrocytes. The results were obtained by phosphor imaging for groups of 3 monkeys immunized with pFVORII and pFVORII/P2P30 or control. The $50 \%$ EBA-175 RII reciprocal blocking titers were calculated based on the binding activity in the presence of pooled sera from the negative control monkeys (data not shown). The geometric mean (unfilled square $(\bullet)$ and standard error of the mean (error bars) are shown. Filled circles $(\bullet)$ represent individual reciprocal blocking titers at $50 \%$.

titers at $50 \%$ blocking are shown in Figure 4 as well as the geometric mean titers for prechallenge and postchallenge sera.

Immunization and P. falciparum Challenge of Aotus monkeys: Boosting of EBA-175 RII Antibodies After Challenge

The course of parasitemias in Aotus monkeys immunized with pFVORII, pFVORII/P2P30, and empty vector after challenge with P. falciparum-infected Aotus erythrocytes is shown in Figure 5. All of the monkeys

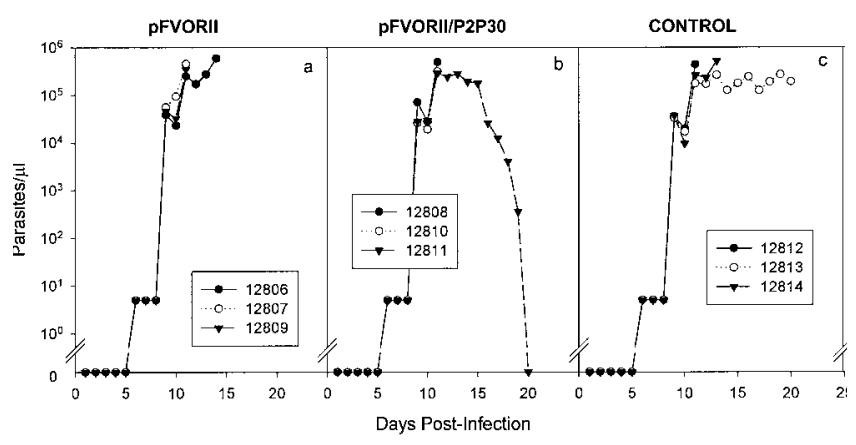

Fig. 5. The course of parasitemias in Aotus monkeys challenged intravenously with $10^{4} P$. falciparum-infected Aotus erythrocytes. Results shown include groups of three monkeys immunized with pFVORII (a), pFVORII/P2P30 that also contains the universal T-cell epitopes P2P30 (b), or empty vector (c). Legends show individual Aotus identifiers for within each group and resultant parasitemias. Monkeys were treated when the parasite density reached $400,000 / \mu l$. except one (Aotus 12811) required treatment because the threshold for total number of parasites per microliter of whole blood was exceeded. Although the parasitemia of Aotus 12811 immunized with pFVORII/P2P30 approached the threshold level for treatment, the animal controlled and cleared the challenge infection. Aotus 12811 was treated on day 60 with mefloquine. An important finding arising from the Aotus study was that the antibody response against EBA-175 RII, including blocking antibodies, was significantly boosted (approximately 10 -fold) by exposure to blood stage infection (Figures 2 and 4 ).

\section{Discussion}

The interaction between the P. falciparum merozoite ligand EBA-175 and its erythrocyte receptor glycophorin A is extremely specific and is a prerequisite for invasion (3). The identification of RII as the receptor-binding domain of EBA-175 has allowed for the development of strategies to target this ligand-receptor interaction in a specific and efficient manner. In the studies described herein, we proved the hypothesis that anti-RII antibodies can effectively block the binding of native EBA-175 to erythrocytes and inhibit merozoite invasion of erythrocytes. We also showed that immunization with a DNA plasmid encoding RII may protect against fulminant disease in the Aotus model. Furthermore, we also produced data suggesting that in the field, vaccine-primed anti-RII antibody responses will be boosted by natural exposure to parasites, thereby enhancing vaccine-induced protective immunity.

Optimal binding of RII to glycophorin A requires Neu5Ac (alpha 2-3) Gal-determinants on O-linked tetrasaccharides displayed on the peptide backbone of glycophorin A (3). Glycophorin B, which has 11 of the $15 \mathrm{O}$-linked tetrasaccharides of glycophorin A at the NH2-terminal half of the extracellular domain, cannot support efficient binding (3). Also, desialated glycophorin A that only displays the peptide backbone cannot support the binding of RII. In competitive binding assays of EBA-175 to erythrocytes, Neu5Ac (alpha2-6) Gal-linkages were only slightly inhibitory (14). Further, even the forms of sialic acids confer specificity because $P$. falciparum invasion of murine erythrocytes requires the Neu5Ac but not the Neu9Ac2 found in some murine strains (15). With such fine specificity and strict requirements for optimal receptor-ligand interaction, one can argue that interference provided by specific antibodies against RII would easily abrogate the binding and thus invasion of merozoites into erythrocytes. This was not the case in vivo with the antibody titers induced in our present Aotus study.

We propose several reasons for this. The simplest explanation is that the levels of antibodies raised by the DNA vaccines were not high enough. 
We now have data indicating that we can increase antibody titers 10 - to 20 -fold by boosting the DNAprimed monkeys with recombinant RII protein in adjuvant or by immunizing with recombinant RII protein alone in adjuvant (22). Another explanation involves the fact that it is not clear whether EBA175 plays as significant role in P. falciparum invasion of Aotus erythrocytes as we believe it does in invasion of human erythrocytes. Aotus sp. are not a natural host for $P$. falciparum. The interaction between Aotus erythrocytes and RII has not been characterized. The sialic acid forms, as well as glycophorins on Aotus erythrocytes, are unknown. The genes encoding the orthologs of human glycophorins in Aotus have neither been identified nor characterized. Orlandi et al. (16) reported the binding of native EBA-175 to Aotus erythrocytes, but his studies showed that this binding is far less efficient than is the binding of EBA-175 RII to human erythrocytes. In fact, in additional studies, we have seen that the binding of EBA-175 RII to Aotus erythrocytes under standard binding assay conditions is not different than its binding to rabbit and guinea pig erythrocytes, erythrocytes that are not permissive to invasion by $P$. falciparum (3,Sim, unpublished). Thus, invasion of $P$. falciparum merozoites into Aotus erythrocytes may be by an alternative mechanism. If EBA-175 does not play a critical role in P. falciparum merozoite invasion of Aotus erythrocytes, then the anti-parasite activity that we have documented is almost certainly a reflection of recognition of surface accessible EBA-175 on merozoites and resultant inhibition of invasion. Thus, we believe that the antiparasite activity in humans of anti-EBA- 175 region II antibodies will probably be greater than in Aotus because EBA-175 plays a crucial role in invasion of erythrocytes in humans.

It is important to highlight the fact that when Aotus monkeys primed by immunization with the DNA vaccines were exposed to $P$. falciparum infection there was significant boosting of the titers of anti-RII antibodies and the titers of antibodies that inhibited binding of EBA-175 to erythrocytes (Figures 2 and 4). We believe these findings are important for two reasons. First, they suggest that in the field immune responses primed by immunization with RII vaccines will be boosted by natural exposure to infection, and these boosted antibody responses may further limit and control infection. Second, these data suggest that boosting of DNA-primed individuals with purified recombinant EBA-175 may lead to significantly enhanced antibody responses as has been the case in other systems (17).

Although RII comprises 616 amino acids, we were concerned that it might not contain adequate CD4+ T-cell epitopes to provide help for production of antibodies in individuals of diverse genetic backgrounds. We, therefore, constructed a plasmid expressing only RII and another one expressing RII fused to P2P30, which contains universal T-cell epitopes from tetanus toxin (9) to ask if in preclinical models P2P30 would boost antibody titers. The two DNA plasmids were equally immunogenic in all five inbred strains of mice, and in small numbers of Aotus monkeys, indicating that the P2P30 epitopes not only were not required for $\mathrm{T}$ cell help, but did not boost significantly the antibody titers generated.

One concern regarding anti-RII vaccines has been whether they will be effective against all strains of P. falciparum. Some strains of P. falciparum use an alternative pathway for invasion of erythrocytes. These strains do not require sialic acids on glycophorin A to bind and invade as they have been shown to invade neuraminidase treated erythrocytes $(18,19,20)$. However, we had previously shown great conservation of the primary sequence of RII among strains of P. falciparum (4). Also, EBA-175 is expressed in all $P$. falciparum strains examined to date (4, data not shown). Further, it has been shown previously that antibodies against RII can block the invasion of merozoites into erythrocytes by merozoites of a $P$. falciparum strain that does not require sialic acids for invasion (21).

One monkey controlled its infection, a very unusual event in Aotus lemurinus lemurinus infected with FVO. However, there was no single assay that clearly predicted this outcome, and there was no protection in other monkeys. This may have been owing to suboptimal immunogenicity, and as described above, we have subsequently shown that we can induce much better antibody titers. Given our reservations regarding the interaction between EBA-175 and Aotus erythrocytes, and our finding of excellent boosting of EBA-175 DNA vaccine primed antibodies, it may be that the appropriate next step is evaluation of the potential of this vaccine candidate in humans.

\section{Acknowledgments}

We thank Trevor R. Jones (Malaria Program, NMRC) for assistance with the statistical analysis and Peter Hobart (Vical Inc., San Diego, CA) for providing the DNA vaccine plasmids. The experiments reported here were conducted according to the principles set forth in the "Guide for the Care and Use of Laboratory Animals," Institute of Laboratory Animals Resources, National Research Council, National Academy Press, 1996. This work was funded by: a Phase II NIAID/ NIH Small Business Innovative Research Grant and supplement: AI36758 awarded to BKLS, a UNDP/ World Bank/WHO/TDR\#931180 awarded to BKLS, the Naval Medical Research Center work unit \#62787A.870.F.A0010, and a grant to Gorgas Memorial Laboratory from the U.S. Army, Contract DAMD17-91-C-1072.

The opinions and assertions herein are the private ones of the authors and are not to be construed as official or as reflecting the views of the U.S. Navy or the U.S. Department of Defense. 


\section{References}

1. Camus D, Hadley TJ. (1985) A Plasmodium falciparum antigen that binds to host erythrocytes and merozoites. Science 230: 553-556.

2. Sim BKL, Orlandi PA, Haynes JD, et al. (1990) Primary structure of the 175K Plasmodium falciparum erythrocyte binding antigen and identification of a peptide which elicits antibodies that inhibit malaria merozoite invasion. J. Cell Biol. 111: 1877-1884.

3. Sim BKL, Chitnis CE, Wasniowska K, Hadley TJ, Miller LH. (1994) Receptor and ligand domains for invasion of erythrocytes by Plasmodium falciparum. Science 264: 1941-1944.

4. Liang H, Sim BKL. (1997) Conservation of structure and function of the erythrocyte-binding domain of Plasmodium falciparum EBA-175. Mol. Biochem. Parasitol. 84: 241-245.

5. Vernes A, Haynes JD, Tapchaisri P, Williams JL, Dutoit E, Diggs CL. (1984) Plasmodium falciparum strain-specific human antibody inhibits merozoite invasion of erythrocytes. Am. J. Trop. Med. Hyg. 33: 197-203.

6. Aley SB, Sherwood JA, Howard RJ. (1984) Knob-positive and knob-negative Plasmodium falciparum differ in expression of a strain-specific malarial antigen on the surface of infected erythrocytes. J. Exp. Med. 160: 1585-1590.

7. Becker SI, Wang R, Hedstrom RC, et al. (1998) Protection of mice against Plasmodium yoelii sporozoite challenge with $P$. yoelii merozoite surface protein 1 DNA vaccines. Infect. Immun. 66: 3457-3461.

8. Hartikka J, Sawdey M, Cornefert-Jensen F, et al. (1996) An improved plasmid DNA expression vector for direct injection into skeletal muscle. Hum. Gene Ther. 7: 1205-1217.

9. Valmori D, Sabbatini A, Lanzavecchia A, Corradin G, Matricardi PM. (1994) Functional analysis of two tetanus toxin universal $\mathrm{T}$ cell epitopes in their interaction with DR 1101 and DR 1104 alleles. J. Immunol. 152: 2921-2929.

10. Ma NS, Rossan RN, Kelley ST, Harper JS, Bedard MT, Jones TC. (1978) Banding patterns of the chromosomes of two new karyotypes of the owl monkey, Aotus captured in Panama. J. Med. Primatol. 7: 146-155.

11. Obaldia, ND. (1991) Detection of Klebsiella pneumoniae antibodies in Aotus l. lemurinus (Panamanian owl monkey) using an enzyme linked immunosorbent assay (ELISA) test. Lab. Anim. 25: 133-141.

12. Thomas AW, Trape JF, Rogier C, Goncalves A, Rosario VE, Narum DL. (1994) High prevalence of natural antibodies against Plasmodium falciparum 83-kilodalton apical membrane antigen (PF83/AMA-1) as detected by capture-enzymelinked immunosorbent assay using full-length baculovirus recombinant PF83/AMA-1. Am. J. Trop. Med. Hyg. 51: 730-740.

13. Liang H, Narum DL, Fuhrmann SR, Luu T, Sim BKL. (2000) A recombinant baculovirus-expressed Plasmodium falciparum receptor-binding domain of erythrocyte binding protein EBA-175 biologically mimics native protein. Infect. Immun. 68: 3564-3568.

14. Orlandi PA, Klotz FW, Haynes JD. (1992) A malaria invasion receptor, the 175-kilodalton erythrocyte binding antigen of Plasmodium falciparum recognizes the terminal Neu5Ac(alpha 2-3)Gal-sequences of glycophorin A. J. Cell Biol. 116: 901-909.

15. Klotz FW, Orlandi PA, Reuter G, et al. (1992) Binding of Plasmodium falciparum 175-kilodalton erythrocyte binding antigen and invasion of murine erythrocytes requires $\mathrm{N}$-acetylneuraminic acid but not its $\mathrm{O}$-acetylated form. Mol. Biochem. Parasitol. 51: 49-54.

16. Orlandi PA, Sim BKL, Chulay JD, Haynes JD. (1990) Characterization of the 175-kilodalton erythrocyte binding antigen of Plasmodium falciparum. Mol. Biochem. Parasitol. 40: 285-294.

17. Letvin NL, Montefiori DC, Yasutomi Y, et al. (1997) Potent, protective anti-HIV immune responses generated by bimodal HIV envelope DNA plus protein vaccination. Proc. Natl. Acad. Sci. USA. 94: 9378-9383.

18. Dolan SA, Miller LH, Wellems TE. (1990) Evidence for a switching mechanism in the invasion of erythrocytes by Plasmodium falciparum. J. Clin. Invest. 86: 618-624.

19. Dolan SA, Proctor JL, Alling DW, Okubo Y, Wellems TE, Miller LH. (1994) Glycophorin B as an EBA-175 independent Plasmodium falciparum receptor of human erythrocytes. Mol. Biochem. Parasitol. 64: 55-63.

20. Okoyeh JN, Pillai CR, Chitnis CE. (1999) Plasmodium falciparum field isolates commonly use erythrocyte invasion pathways that are independent of sialic acid residues of glycophorin A. Infect. Immun. 67: 5784-5791.

21. Narum DL, Haynes JD, Fuhrmann S, et al. (2000) Antibodies against the Plasmodium falciparum receptor binding domain of EBA-175 block invasion pathways that do not involve sialic acids. Infect. Immun. 68: 1964-1966.

22. Jones TR, Narum DL, Gozalo AS, et al (2001) Protection of Aotus monkeys by Plasmodium falciparum EBA-175 region II DNA Prime-Protein boost immunization regimen. J. Infect. Dis. 183: 303-312. 